\title{
A NOTE ON GRÜSS TYPE INEQUALITIES VIA CAUCHY'S MEAN VALUE THEOREM
}

\author{
B. G. PACHPATTE
}

Abstract. The main aim of the present note is to establish two new weighted Grüss type integral inequalities by using a fairly elementary analysis.

Mathematics subject classification (2000): 26D15, 26D20.

Key words and phrases: Grüss type inequalities, Cauchy's mean value theorem, weighted integral inequalities, integrable function.

\section{REFERENCES}

[1] S. S. DRAGomiR, Some integral inequalities of Grüss type, Indian J. Pure and Appl. Math. 31(2000), 379-415.

[2] S. S. DRAGOMIR, Some Ostrowski type inequalities via Cauchy's mean value theorem, RGMIA Research Report Collection (Supl.) 6(2003), 1-11, Art. 9.

[3] A. M. FInK, A treatise on Grüss inequality, Analytic and Geometric Inequalities and Applications, T. M. Rassias and H. M. Srivastava (eds.), Kluwer Academic Publishers, Dordrecht 1999, 93-113.

[4] G. GRÜss, Über das maximum des absoluten Betrages von

$$
\frac{1}{b-a} \int_{a}^{b} f(x) g(x) d x-\frac{1}{(b-a)^{2}} \int_{a}^{b} f(x) d x \int_{a}^{b} g(x) d x,
$$

Math. Z. 39(1935), 215-226.

[5] D. S. Mitrinović, J. E. PEČARIĆ AND A. M. FinK, Classical and new inequalities in Analysis, Kluwer Academic Publishers, Dordrecht, 1993.

[6] B. G. PachPatte, On Grüss type inequalities for double integrals, J. Math. Anal. Appl. 267(2002), 454-459.

[7] B. G. PachPatte, On Grüss type integral inequalities, J. Inequal. Pure and Appl. Math. 3(1)(2002), Art. 11.

[8] B. G. PACHPATte, New weighted multivariate Grüss type inequalities, J. Inequal. Pure and Appl. Math. 4(5) (2003), Art. 108.

[9] B. G. Pachpatte, On Trapezoid and Grüss like integral inequalities, Tamkang J. Math. 34(2003), 365-369.

[10] W. Rudin, Principles of Mathematical Analysis, McGraw-Hill Book Company, Inc. 1953. 